\title{
Spatiotemporal parameters of gait during treadmill and overground walking in Parkinson's disease
}

\author{
Olalla Bello, José Andrés Sánchez, Cristina Vázquez-Santos and Miguel Fernández- \\ Del-Olmo
}

\begin{abstract}
Despite the increasing number of studies that have examined the therapeutic effect of treadmill training programs in Parkinson's disease (PD), there has been little research to evaluate the modifications of gait induced by treadmill walking. We investigated spatiotemporal differences between treadmill and overground walking in patients with PD. PD patients significantly increased their step length and step height; and reduced their cadence, step width and step width variability on the treadmill in comparison with walking overground. PD patients are able to attenuate their short shuffling steps when walking on a treadmill.
\end{abstract}

Keywords

Gait, Parkinson's disease, rehabilitation

\section{Introduction}

Gait in PD classically presents a reduction in stride length, and an increase in double support phase and stride-to-stride variability [1, 2]. People with PD also exhibit a slow, shuffling and forward-stooped gait [3]. Shuffling is produced when feet barely leave the ground and this gait abnormality has been related with the quality of life in PD [4].

In the last few years, an increasing number of studies have examined the therapeutic effect of treadmill training on gait in PD (for review see 5 and 6) [5, 6]. Several studies have examined gait kinematic differences between treadmill and overground walking in PD, reporting a reduced gait variability [7] and an increased step length [8,9] during treadmill walking. However, it remains unknown whether other biomechanical parameters, such as step width and step height, are modified during treadmill walking in PD. A better understanding of the modification of the gait parameters may help to explain the potential benefits of treadmill training in PD. Thus, the main goals of this study were to compare spatiotemporal parameters of gait in PD patients during treadmill and overground walking, and to determine how these changes compare to those seen in age matched healthy controls.

\section{Materials and methods}

Eight subjects with PD in stage 3 of the Hoehn and Yahr classification [10] (4 males and 4 females; mean age 65.56 years, range 52-74 years) and eight age-matched healthy controls ( 4 males and 4 females, mean age 63.24 years, range 51-74 years) were recruited. Exclusion criteria included inability to walk unassisted for 10 minutes, dementia, other neurological disorders; and visual, respiratory, orthopaedic or cardiovascular problems that affect gait. The level of functional disability was examined using the Unified Parkinson's Disease Rating Scale [11] and the Schwab and England scale [12]. Data collection was carried out with the subjects in "on" medication state. All participants provided informed consent according to the declaration of Helsinki and the Ethics Committee of our Institution approved the experimental protocol (2009/346).

The testing procedure has been previously described [9]. Briefly, each subject was evaluated walking overground a distance of $60 \mathrm{~m}$ on a $10 \mathrm{~m}$ walkway and after that was tested on the treadmill (SporsArt 6300, Sports Arts Fitness, China) for 1 minute at that same speed of overground walking. A familiarization with the testing conditions was performed before data was collected. All participants 
walked on the treadmill while holding the handrails and with a safety harness, which did not support the subject's body weight.

Four video cameras (Sony DCR-HC19E, Sony Corporation, Japan) were used to register gait performance in the transverse, sagittal, and coronal plane $(50 \mathrm{~Hz})$. The recording volume was $2.5 \mathrm{~m}$ long, $1 \mathrm{~m}$ wide, and $0.52 \mathrm{~m}$ high for the overground walking and $1.27 \mathrm{~m}$ long, 0.46 wide, and $0.27 \mathrm{~m}$ high for the treadmill gait. A retroreflective marker $(0.022 \mathrm{~m}$ in diameter) was placed on the posterior part of the heel on each shoe and its coordinates were obtained using Kinescan software 2001 (Biomechanics Institute of Valencia, Spain). Two photoelectric cells (Omron E3G-R17, Omron Corporation, Japan) were used during the overground tests to calculate gait speed.

The variables measured included: step length $(\mathrm{m})$ as the anterior-posterior distance from heel-strike of one foot to heel-strike of the contralateral foot; step width $(\mathrm{m})$ as the medial-lateral distance between those locations; step height $(\mathrm{m})$ as the maximum vertical dis- placement of the heel during the swing phase; cadence (steps/min); coefficient of variation (CV) of step length (\%); CV of step width (\%); and $\mathrm{CV}$ of step height $(\%)$. CV was calculated as follows: (standard deviation / mean) $\times 100$.

We employed the Student's t tests to compare PD and control subjects with respect to background characteristics. A repeated-measures analysis of variance (ANOVA) was carried out to compare overground and treadmill gait using condition (overground and tread-mill) and group (PD and control) as factors. Post hoc tests were computed using the Bonferroni correction. All statistical analyses were performed using PASW Statistics 18 (SPSS Inc, USA). A $P$ value $\leq 0.05$ was considered statistically significant.

\section{Results}

Characteristics of the PD and control group are shown in Table 1. No differences were found for age, weight, leg length or height between groups.

Table 1. Subject characteristics

\begin{tabular}{lccc}
\hline & PD group & Control group & Group differences* \\
\hline & & & \\
Age (years) & $65.56(7.69)$ & $63.24(8.06)$ & NS \\
Weight (Kg) & $72.86(10.25)$ & $73.20(7.51)$ & NS \\
Leg length (cm) & $83.32(5.37)$ & $85.58(3.51)$ & NS \\
Height (cm) & $160.90(7.00)$ & $165.24(7.25)$ & NS \\
Disease duration (years) & $8.33(4.25)$ & - & - \\
UPDRS & $65.43(14.80)$ & - & - \\
S\&E & $72.86(7.56)$ & - & - \\
& & & \\
\hline
\end{tabular}

Data reflect means with standard deviations in parentheses. *Significance was assessed by $t$ test $(P \leq 0.05)$. UPDRS: Unified Parkinson's Disease Rating Scale. S\&E: Schwab and England scale

Table 2 summarizes the main results. The ANOVA showed a significant condition*group interaction in step length $(\mathrm{F} 1,15=15.57, P=0.002)$, cadence $(\mathrm{F} 1,15=14.33, P=0.002)$ and step height $(\mathrm{F} 1,15$ $=8.46, P=0.012)$ without a significant main effect for condition. Post hoc tests showed that for PD subjects, treadmill walking significantly increased step length $(\mathrm{P}=0.007)$ and step height $(\mathrm{P}=0.013)$, and reduced cadence $(P=0.005)$ in comparison with the overground condition. In the control group, there were no significant differences in these variables. There was a significant main effect for condition in step width $(\mathrm{F} 1,15=5.62, P=0.034)$ and $\mathrm{CV}$ of step width $(\mathrm{F} 1,15=6.20, P=0.027)$ without a condition*group interaction. That is, step width and $\mathrm{CV}$ of step width were greater in the overground condition $(\mathrm{M}=0.12, \mathrm{SD}=0.02$ for step width; $\mathrm{M}=18.57, \mathrm{SD}=8.12$ for $\mathrm{CV}$ of step width) than in the treadmill condition in both groups $(M=0.10, S D=0.03$ for step width; $M=13.63, S D=10.50$ for $C V$ of step width). 
Table 2. Means of gait parameters with standard deviations in parenthesis and summary of statistical results

\begin{tabular}{|c|c|c|c|c|c|c|c|c|c|}
\hline & \multicolumn{3}{|c|}{ PD GROUP } & \multicolumn{3}{|c|}{ Control group } & \multicolumn{3}{|c|}{ Anova results $(\mathrm{P})$} \\
\hline & Overground & Treadmill & $\begin{array}{c}\text { Post hoc } \\
\text { test }(\mathrm{P})\end{array}$ & Overground & Treadmill & $\begin{array}{c}\text { Post hoc } \\
\text { test (P) }\end{array}$ & Group & $\begin{array}{c}\text { Conditio } \\
n\end{array}$ & $\begin{array}{l}\text { Group } \times \\
\text { condition }\end{array}$ \\
\hline Step length (m) & $0.46(0.08)$ & $0.53(0.11)$ & 0.007 & $0.71(0.06)$ & $0.67(0.07)$ & 0.130 & $<0.001$ & 0.139 & 0.002 \\
\hline Cadence (steps/min) & $\begin{array}{l}101.15 \\
(10.51)\end{array}$ & $87.40(11.57)$ & 0.005 & $\begin{array}{l}113.26 \\
(10.30)\end{array}$ & $\begin{array}{l}117.59 \\
(17.37)\end{array}$ & 0.226 & 0.004 & 0.070 & 0.002 \\
\hline Step height (m) & $0.21(0.04)$ & $0.24(0.03)$ & 0.013 & $0.26(0.02)$ & $0.25(0.05)$ & 0.424 & 0.062 & 0.115 & 0.012 \\
\hline Step width (m) & $0.12(0.03)$ & $0.10(0.03)$ & - & $0.11(0.02)$ & $0.10(0.03)$ & - & 0.621 & 0.034 & 0.664 \\
\hline CV step length (\%) & $6.79(2.48)$ & $6.70(2.92)$ & - & $3.30(0.37)$ & $6.24(4.87)$ & - & 0.154 & 0.141 & 0.119 \\
\hline CV step height (\%) & $7.82(6.69)$ & $8.73(10.27)$ & - & $3.20(1.13)$ & $2.85(3.35)$ & - & 0.130 & 0.796 & 0.553 \\
\hline CV step width (\%) & $\begin{array}{l}19.33 \\
(9.23)\end{array}$ & $17.83(12.71)$ & - & $17.70(7.26)$ & $8.82(4.27)$ & - & 0.230 & 0.027 & 0.100 \\
\hline
\end{tabular}

Bold values indicate significant differences based on ANOVA or Post hoc analysis

\section{Discussion}

In PD, walking on the treadmill leads to a significant increase in stride length and a decrease in cadence in comparison with overground walking, while no differences were found between both conditions in controls. These results support previous findings that showed step length modifications on the treadmill in PD $[8,9]$. These beneficial biomechanical changes could be the origin of the carryover effects of treadmill walking previously described in PD [13-15].

Only the patients with PD increased their step height when they walked on the treadmill in comparison with overground walking. This result suggests that on the treadmill patients demonstrate a gait pattern with less shuffling than that observed overground. This finding is of importance since shuffling can lead to an increase in falls [16]. To our knowledge, this is the first study in PD that evaluates the effects of treadmill walking on step height. The mechanisms underlying the improvement in step length and step height in PD during treadmill walking remain largely unknown. Whilst walking on a treadmill the subjects had a hand support and a stable visual feedback, which could account for these improvements. However, a recent study has suggested that improvement in the step length is due to the proprioceptive information generated by the belt movement, as no improvement was reported when patients used a treadmill simulator which did not have a belt [8]. Therefore, when walking on the treadmill, this pro- prioceptive information could act as a sensory cue, allowing PD patients to use neuronal circuits different from those of the basal ganglia-supplementary motor area pathway [17] and diminishing motor symptoms such as shuffling gait. Another possible hypothesis is that the treadmill could activate central pattern gen- erators by providing different sensory inputs to the subject (e.g. hip extension that could promote hip flex- ion) [18]. Therefore, both mechanisms could account for the changes of step height and step length reported in our study.

Both PD and control subjects showed reduced step width and variability when walking on the treadmill in comparison with walking overground. Alterations in these parameters have been associated with falls in older people $[19,20]$. The increments in step width and step width variability could reflect a strategy of the central nervous system to control the center of mass and maximize stability [21]. Therefore, our results could suggest an improvement in balance while walking on the treadmill, mainly due to the fact that subjects kept their hands on the handrails, which could help to improve their stability and consequently reduce step width and step width variability.

In conclusion, our study shows that patients with PD can improve their gait pattern when walking on a tread- mill compared with overground gait, as illustrated by longer step length, smaller step width, decreased step width variability and higher foot elevation. This is of relevance since gait with short shuffling steps is a significant challenge that affects patients in advanced state of the disease. Further controlled studies should investigate long-term effects of a treadmill walking program on these gait parameters. 


\section{Acknowledgments}

This work was supported by the Ministerio de Ciencia e Innovación (DEP2011-22466), Spain. We thank Susana Grueiro and Noa Fogelson for the revision of the manuscript.

\section{Conflict of interest}

The authors have no conflict of interest to report.

\section{References}

[1] Azulay JP, Mesure S, Amblard B, Blin O, Sangla I, \& Pouget J (1999) Visual control of locomotion in Parkinson's disease. Brain , 122, 111-120.

[2] Hausdorff JM, Cudkowicz ME, Firtion R, Wei JY, \& Goldberger AL (1998) Gait variability and basal ganglia disorders: Stride-to-stride variations of gait cycle timing in Parkinson's disease and Huntington's disease. Mov Disord , 13 , 428-437.

[3] Knutsson E (1972) An analysis of Parkinsonian gait. Brain , 95 , 475-486.

[4] Rahman S, Griffin HJ, Quinn NP, \& Jahanshahi M (2008) Quality of life in Parkinson's disease: The relative importance of the symptoms. Mov Disord , 23, 1428-1434

[5] Herman T, Giladi N, \& Hausdorff JM (2009) Treadmill training for the treatment of gait disturbances in people with Parkinson's disease: A mini-review. J Neural Transm , 116, 307-318.

[6] Mehrholz J, Friis R, Kugler J, Twork S, Storch A, \& Pohl M (2010) Treadmill training for patients with Parkinson's disease. Cochrane Database Syst Rev , CD007830.

[7] Frenkel-Toledo S, Giladi N, Peretz C, Herman T, Gruendlinger L, \& Hausdorff JM (2005) Treadmill walking as an external pacemaker to improve gait rhythm and stability in Parkinson's disease. Mov Disord , 20 , 1109-1114.

[8] Bello O, Marquez G, Camblor M, \& Fernandez-Del-Olmo M (2010) Mechanisms involved in treadmill walking improvements in Parkinson's disease. Gait Posture , 32, 118-123.

[9] Bello O, Sanchez JA, \& Fernandez-del-Olmo M (2008) Tread- mill walking in Parkinson's disease patients: Adaptation and generalization effect. Mov Disord , 23 , 1243-1249.

[10] Hoehn MM, \& Yahr MD (1967) Parkinsonism: Onset, progression and mortality. Neurology , 17 , 427-442.

[11] Fahn S, \& Elton RL (1987) Unified Parkinson's Disease Rating Scale. In Recent developments in Parkinson's disease, Fahn S, Marsden CD, Goldstein M, \& Calne DB, eds. Macmillan Healthcare Information, Florham Park, NJ, pp. 153-163.

[12] Schwab RS, \& England AC (1969) Projection technique for evaluating surgery in Parkinson's disease. In Third Symposium on Parkinson's Disease, Gillingham FJ, \& Donaldson IML, eds. E and S Livingstone, Edinburgh, pp. 152-157.

[13] Bello O, Sanchez JA, Lopez-Alonso V, Marquez G, Morenilla L, Castro X, Giraldez M, Santos-Garcia D, \& Fernandez- Del-Olmo M (2013) The effects of treadmill or overground walking training program on gait in Parkinson's disease. Gait Posture , 38 , 590-595.

[14] Fisher BE, Wu AD, Salem GJ, Song J, Lin CH, Yip J, Cen S, Gordon J, Jakowec M, \& Petzinger G (2008) The effect of exercise training in improving motor performance and corticomotor excitability in people with early Parkinson's disease. Arch Phys Med Rehabil , 89, 1221-1229.

[15] Miyai I, Fujimoto Y, Yamamoto H, Ueda Y, Saito T, Nozaki S, \& Kang J (2002) Long-term effect of body weight-supported treadmill training in Parkinson's disease: A randomized con- trolled trial. Arch Phys Med Rehabil , 83, 1370-1373.

[16] Aita JF (1982) Why patients with Parkinson's disease fall. JAMA, 247 , 515-516.

[17] Brooks DJ (1999) Functional imaging studies in Parkinson's disease. In Parkinson's disease The treatment options, Lewitt P, \& Oertel W, eds. Martin Dunitz, London, pp. 21-38.

[18] Bello O, \& Fernandez-Del-Olmo M (2012) How does the treadmill affect gait in Parkinson's disease? Curr Aging Sci , 5 , 28-34.

[19] Brach JS, Berlin JE, VanSwearingen JM, Newman AB, \& Studenski SA (2005) Too much or too little step width variability is associated with a fall history in older persons who walk at or near normal gait speed. $\mathrm{J}$ Neuroeng Rehabil , 2 , 21.

[20] Maki BE (1997) Gait changes in older adults: Predictors of falls or indicators of fear. J Am Geriatr Soc , 45 , 313-320. [21] MacLellan MJ, \& Patla AE (2006) Adaptations of walking pattern on a compliant surface to regulate dynamic stability. Exp Brain Res , 173 , 521-530. 